\title{
Colitis nerviosa en estudiantes de medicina de la Escuela Superior de Tepeji del Río
}

\section{Nervous Colitis in Medical Students of the Tepeji Del Rio high school}

\author{
Wendy Y. González-Maldonado ${ }^{a}$, Nancy Rodríguez-Trejo ${ }^{b}$, Eloísa E. Vargas-Cano ${ }^{c}$, Mauro \\ Nájera-Arenas ${ }^{d}$, Gustavo Doniz-Valdez ${ }^{e}$, Diana V. Sánchez-Martínez ${ }^{f}$.
}

\begin{abstract}
:
This research work consists of the description and analysis of the current conditions of medical students suffering from nervous colitis. The research was carried out at the Escuela Superior de Tepeji del Rio from the first to the fourth semester. The objective of the research was oriented to analyse if Aloe vera infusions are effective for the treatment of nervous colitis, this analysis will help to design an alternative treatment substituting drugs for Aloe infusions.

The research was carried out based on the case methodology, obtaining information from surveys carried out on 136 medical students, of which $43.3 \%$ presented nervous colitis. The analyzed data suggested that the students present differences in the signs and symptoms they present, preventive strategies are described: such as promoting physical exercise, following a balanced diet; although there is no solid scientific evidence, these interventions can improve symptoms and therefore the quality of life of specific patients.
\end{abstract}

Keywords:

Nervous colitis, aloe vera, infusions, preventive strategies, balance diet.

\section{Resumen:}

El presente trabajo de investigación consiste en la descripción y análisis de las condiciones actuales de los estudiantes de medicina que padecen colitis nerviosa. La investigación se realizó en la Escuela Superior de Tepeji del Río de primer a cuarto semestre. El objetivo de la investigación se orientó a analizar las infusiones de Aloe vera como tratamiento de colitis nerviosa, dicho análisis, ayudará diseñar una alternativa de tratamiento sustituyendo fármacos por infusiones de Aloe. La investigación se realizó en base a la metodología del caso, obteniendo la información de encuestas realizadas a 136 estudiantes de medicina, de los cuales el $43.3 \%$ presentó colitis nerviosa. Los datos analizados sugieren que los estudiantes expresan diferencias en los signos y síntomas que presentan. Se describen estrategias preventivas: como el promover la realización de ejercicio físico, seguir una dieta equilibrada; aunque no se dispone de evidencia científica sólida, estas intervenciones pueden mejorar los síntomas y por tanto la calidad de vida de determinados pacientes.

\section{Palabras Clave:}

Colitis nerviosa, aloe vera, infusiones, estrategias preventivas, dieta equilibrada.

\section{Planteamiento del problema}

La colitis nerviosa es una enfermedad crónica benigna que lo padece entre 16 y $30 \%$ de la población en México según el instituto mexicano del seguro social. Esto puede ser por el desorden en los cambios alimenticios, factores ambientales o emocionales, agentes infecciosos como virus, bacterias, amibas, trastornos en la dieta, etc. Comúnmente hablamos de colitis nerviosa, sin embargo, el término exacto para esta patología es Síndrome de

\footnotetext{
${ }^{a}$ Universidad Autónoma del Estado de Hidalgo, https://orcid.org/0000-0003-1000-020X, Email: go413637@uaeh.edu.mx

b Universidad Autónoma del Estado de Hidalgo, https://orcid.org/0000-0001-5233-9530, Email: ro413642@uaeh.edu.mx

${ }^{c}$ Autor de correspondencia, Universidad Autónoma del Estado Hidalgo, https://orcid.org/0000-0001-5424-5124, Email: va335035@uaeh.edu.mx

${ }^{d}$ Universidad Autónoma del Estado de Hidalgo, https://orcid.org/0000-0003-4540-9881, Email: na414677@uaeh.edu.mx

${ }^{e}$ Universidad Autónoma del Estado de Hidalgo, https://orcid.org/0000-0001-8409-4451, Email: do413566 @uaeh.edu.mx
}

u Uiversidad Autónoma del Estado de Hidalgo, https://orcid.org/0000-0002-7660-7234, Email: 
Intestino Irritable o por sus siglas en inglés abreviado como SII.

Se define entonces el Síndrome del Intestino Irritable como una de las afecciones gastrointestinales más comunes que puede adquirir cualquier individuo, sin embargo, investigaciones previas demuestran que en los estudiantes de medicina es más recurrente (Andrades $\mathrm{A}$. 2017).

De acuerdo a lo anterior el enfoque principal de esta investigación se basa en los síntomas psicofisiológicos subjetivos del Síndrome del Intestino Irritable, es por ello que se llevó a cabo la recolección de información en los meses septiembre- octubre 2019 a los estudiantes de la Licenciatura de Médico Cirujano en la Escuela Superior de Tepeji del Río perteneciente a la Universidad Autónoma del Estado de Hidalgo.

En cifras generales, el SII en México lo padecen con mayor frecuencia mujeres de 25 a 40 años. El $75 \%$ de los pacientes no acude al médico porque considera que solo se presenta al sufrir estrés o ansiedad. Más de $20 \%$ de los mexicanos padece síndrome de intestino irritable (Mendoza M.2013).

Si comparamos las cifras anteriores con las obtenidas a nivel del estado de Hidalgo la variación es notablemente diferente. De acuerdo al Instituto de Seguridad y Servicios Sociales de los Trabajadores del Estado (ISSSTE) ubicado en Hidalgo, aseguró que, del total de pacientes atendidos en una semana, el promedio de los que presentan colitis nerviosa es de $20 \%$, aproximadamente, y aqueja a pacientes desde los 12 años hasta 65 años (Mayo Clinic 15 de agosto, 2019).

Estos datos no suelen ser de preocupación, pero como se menciona en cifras, este síndrome afecta tanto a mujeres como a hombres sin importar la edad, sin embargo, existe una prevalencia aún mayor en el ámbito estudiantil, pues las tasas de morbilidad de padecerla aumentan, debido a factores tanto ambientales, sociales y por supuesto, biológicos.

Los estudiantes son más propensos a padecer SII a lo largo de la carrera universitaria; en específico, los estudiantes de la Licenciatura de Médico Cirujano (Mendoza. M, 2013) pues la mayoría no lleva una dieta adecuada o balanceada, es por eso que se plantea la propuesta de una alternativa de tratamiento natural evitando así el consumo de fármacos que a la larga pueden dañar la mucosa intestinal. Dicha alternativa se basa en la extracción de la pulpa del Aloe vera para posteriormente emplearlo en infusiones.

Durante siglos, el Aloe vera se ha usado por sus propiedades medicinales y terapéuticas desde nuestros antepasados hasta la actualidad, entonces ¿por qué no seguirla utilizando? Diversas investigaciones la han catalogado como "planta medicinal" por sus efectos antiinflamatorios, hipoglucémicos, protectores gástricos y aliviadores de quemaduras y heridas (Andrades A. 2017). Por otra parte, está bien constatado el papel del Aloe vera inhibiendo la proliferación de bacterias, tanto Gram positivas como Gram negativas, así como mejorando enfermedades provocadas por diferentes especies de hongos.

¿Es eficaz la infusión de Aloe vera para el tratamiento de la colitis nerviosa?

\section{Justificación}

La presente investigación se enfocará en analizar el uso del Aloe vera como una alternativa de tratamiento para combatir el padecimiento del Síndrome de Intestino Irritable, cuya enfermedad es una de las más comunes sin distinción de edad, ya que es un trastorno digestivo que se genera por múltiples factores, tanto psicológicos, emocionales, sociales, económicas entre otros.

Así, el presente trabajo permitirá mostrar los resultados obtenidos con estudiantes de la Licenciatura de Médico Cirujano, dado que son más susceptibles debido a las alteraciones dentro de la dieta que se vive a diario unido al estrés. Haciendo hincapié en sus beneficios que trae consigo para la salud física ayudando a problemas de diarrea o estreñimiento, absorción mejor de nutrientes de los alimentos y favoreciendo la excreción de microorganismos dañinos.

\section{Hipótesis}

El Síndrome de Intestino irritable es un trastorno funcional digestivo, se caracteriza por la presencia de dolor abdominal y otros síntomas asociados a las alteraciones del ritmo intestinal, dicho padecimiento comúnmente afecta a los estudiantes de Medicina (Mearin F, Montoro M.).

Con el consumo de infusiones de Aloe vera se reducen en un $70 \%$ los signos y síntomas de la colitis nerviosa (Smith J. (2013).

\section{Objetivo general}

Realizar infusiones a través de la pulpa obtenida del Aloe vera para emplearla como tratamiento alternativo del síndrome de intestino irritable y así registrar su eficacia.

\section{Objetivos específicos}

- Mostrar criterios diagnósticos de síndrome de intestino irritable mediante el conocimiento científico, para la identificación de dicha patología.

- Evaluar la eficacia de la infusión de Aloe vera en estudiantes de medicina de la Escuela Superior de Tepeji del Río para disminuir la colitis nerviosa

- Aplicar encuestas mediante formularios de Google forms a estudiantes de medicina de la escuela superior de Tepeji del Río para identificar sus conocimientos sobre el Aloe vera.

\section{Marco teórico}

\subsection{Historia}

El "Síndrome del Intestino irritable" fue descrito por primera vez en 1812 por el médico inglés William Powell. Tras diversas investigaciones, la historia de esta patología se remonta al siglo XIX (Juárez. 2009). Tales investigaciones son mencionadas en el diagrama 
correspondiente a la Figura 1. Línea del tiempo 1: investigaciones del SII (Juárez, 2009).

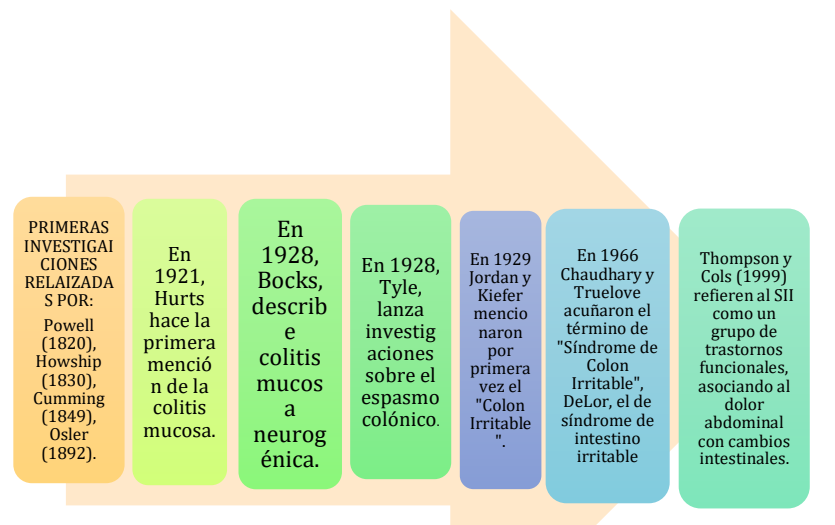

Figura 1. Línea del tiempo 1: investigaciones del SII (Juárez, 2009).

\subsection{Diagnóstico}

A lo largo del tiempo, se han propuesto diversos criterios que permiten establecer un diagnóstico positivo basado en la presencia de síntomas, evitando con ello pruebas superfluas (Otero W., Gómez M. 2015). Desde los criterios iniciales de Manning formulados en 1984, se han realizado distintas propuestas. En los últimos años, la mayoría de los clínicos han utilizado los denominados criterios de Roma por comités de expertos que se reunían periódicamente en Italia (Otero W., Gómez M. 2015). En 1992 se publicaron los criterios de Roma I, en 1999 los criterios de Roma II y en 2006 los criterios de Roma III, los cuales son:

*Dolor o disconfort abdominal recidivante al menos de 3 días durante los últimos 3 meses que se asocia a dos o más de los siguientes casos:

\section{Mejoría en la defecación}

El comienzo se asocia a un cambio en la frecuencia y forma (consistencia) de las deposiciones.

El término disconfort se refiere a una sensación desagradable que no se describe como dolor. Se requiere que aparezca al menos 2 días por semana durante el periodo de cribado (Otero W., Gómez M. 2015).

Los síntomas deben cumplirse al menos durante tres meses y haber comenzado al menos 6 meses antes del diagnóstico.
Tabla 1. Criterios de Roma III para SII (Otero W., Gómez M. 2015).

\begin{tabular}{|l|l|l|}
\hline $\begin{array}{l}\text { SIl con } \\
\text { predominio de } \\
\text { estreñimiento: }\end{array}$ & $\begin{array}{l}\text { Heces duras } \geq 25 \% \text { de las } \\
\text { ocasiones/heces "sueltas" o acuosas } \leq \\
25 \% \text { de las ocasiones. }\end{array}$ \\
\hline $\begin{array}{l}\text { SIl con } \\
\text { predominio de } \\
\text { diarrea: }\end{array}$ & $\begin{array}{l}\text { Heces "sueltas" o acuosas } \geq 25 \% \text { de las } \\
\text { ocasiones/heces duras }<5 \% \text { de las } \\
\text { defecaciones. }\end{array}$ \\
\hline Patrón mixto: & $\begin{array}{l}\text { Heces duras } \geq 25 \% \text { de las } \\
\text { ocasiones/heces "sueltas" o acuosas } \geq \\
\text { 25\% de las ocasiones. }\end{array}$ \\
\hline $\begin{array}{l}\text { Patrón } \\
\text { indefinido: }\end{array}$ & $\begin{array}{l}\text { Anormalidad insuficiente de la consistencia } \\
\text { de las deposiciones para reunir los criterios } \\
\text { expuestos para el resto de las categorías. }\end{array}$ \\
\hline
\end{tabular}

Síntomas que apoyan el diagnóstico pero que no forman parte de los criterios de Roma III son:

a) Frecuencia de las deposiciones $\leq 3$ veces por semana $0>3$ veces por día.

b) Esfuerzo defecatorio excesivo.

c) Urgencia defecatoria.

d) Sensación de evacuación incompleta.

e) Emisión de moco en la deposición.

f) Sensación de hinchazón abdominal.

\subsection{Fisiopatología (SII)}

La patogénesis del SIl es compleja, heterogénea y sólo parcialmente entendida. Múltiples factores probablemente contribuyen para el desarrollo y las manifestaciones clínicas de la entidad (Otero W., Gómez M. (2015). Los mecanismos clásicamente considerados que participan y los niveles de evidencia son:

- Hipersensibilidad visceral: Aproximadamente el 70\% de los pacientes pueden tener disminución del umbral de sensibilidad visceral, pero no somática (Otero W., Gómez M. 2015). Se ha encontrado que el Barostat rectal con una presión umbral de $40 \mathrm{mmHg}$, tiene sensibilidad de $95.5 \%$, especificidad de $72 \%$ y valor predictivo positivo de $90 \%$ para el diagnóstico y discriminación de otras causas de dolor rectal (Otero W., Gómez M. 2015).

-Alteración de la motilidad: las diferentes alteraciones encontradas tienen pobre correlación con los síntomas del paciente.

- Factores Psicosociales: Contribuyen, pero no causan los síntomas.

-Agentes infecciosos: Las infecciones explican del 6-17\% de todos los casos de SII, sin embargo, los del 7 al 33\% de los pacientes con gastroenteritis infecciosas desarrollan SIl en el futuro. Se estima que una infección bacteriana gastrointestinal previa, se asocia con un riesgo de 11 veces de desencadenar SII, comparado con los que la tienen.

- Factores genéticos: la evidencia para la participación de factores genéticos en el SII incluye la frecuencia dos veces mayor en gemelos homocigotos, comparados con heterocigotos, pero la concordancia en los gemelos 
monocigotos no es $1: 1$, indicando que se necesitan otros factores, como los ambientales.

-Serotonina: El 95\% de la serotonina o 5 hidroxitriptamina $(5 \mathrm{HT})$ del cuerpo humano está en el tracto gastrointestinal principalmente en las células enterocromafines $(90 \%)$ y en menor proporción $(10 \%)$ en las neuronas entéricas. Hay por lo menos 14 clases de receptores de $5 \mathrm{HT}$ con diversas funciones tanto en el sistema nervioso central y el periférico. En el SIl los principales receptores son los 5HT3 y 5HT4 (Otero W., Gómez M. 2015).

La acción de la 5 HT termina cuando es removida del espacio intersticial mediante recaptación por acción del transportador de serotonina (Morales, P. 2012). La 5 HT tiene un papel fundamental en la regulación de la motilidad, secreción y sensibilidad del tracto gastrointestinal a través de la activación de numerosos receptores distribuidos ampliamente en los nervios entéricos y aferentes sensoriales. Sus acciones son muy complejas y puede producir contracción del músculo liso al estimular nervios colinérgicos o relajación por estimulación de neuronas inhibidoras que liberan óxido nítrico (Morales, P. 2012). La 5 HT es liberada en la mucosa y en las neuronas sensoriales intrínsecas como las extrínsecas. En las primeras inicia reflejos peristálticos y secretorios a través de receptores $5 \mathrm{HT} 4$ y las últimas modula la sensibilidad vía receptores 5 HT3. Los receptores 5HT4 están localizados sobre las células enterocromafines, musculares lisas, y sobre las neuronas (Kallergis, K. 2017). La liberación de 5HT de las células enterocromafines, es uno de los iniciadores de la peristalsis a través de los $5 \mathrm{HT} 4$ sobre los aferentes primarios. Los receptores 5 HT3 están distribuidos sobre las neuronas motoras entéricas, terminales periféricas de aferentes viscerales y en el sistema nervioso central (centro del vómito). El antagonismo de esos receptores reduce el dolor visceral, el tránsito colónico y las secreciones del Intestino delgado (Kallergis, K. 2017).

En los años 90 apareció la mención de la sensibilidad visceral y la motilidad intestinal mediada por 5 hidroxitriptamina (SHT), substancia cuya distribución es $5 \%$ en el sistema nervioso central (SNC) y $95 \%$ en el tracto gastrointestinal (células enterocromafines y neuronales). La $5 \mathrm{HT}$ tiene efectos intrínsecos aferentes (control de los reflejos y la motilidad gastrointestinal) y extrínsecos, proyectados en el SNC y es mediadora en la percepción visceral del dolor (Kallergis, K. 2017). El sistema nervioso autónomo, a través del parasimpático, el simpático y del sistema nervioso entérico, tiene participación, y especialmente este último que contiene muchos neurotransmisores, incluida la 5hidroxitriptamina. Estos mediadores son los responsables de la respuesta motora y secretora que caracterizan al SII (Hernández, N. 2011).

\subsection{Epidemiología}

Es muy frecuente en la población en general y constituye una de las primeras causas de consultas (Mendoza R. 2013). Aunque su prevalencia varía de acuerdo a la población estudiada oscila entre el 5-15\%, en dependencia de los criterios empleados para el diagnóstico; $5 \%$ con predominio del sexo femenino (relación hombre/ mujer 1:2), esto se relaciona con la disminución importante en la calidad de vida (Hernández, N. 2011). Aunque solo el $15 \%$ de los afectados solicita atención médica, de estos un 40 a 60\% tienen síntomas psicológicos de depresión, ansiedad o ambos (Bautista R., Ortiz R., Muñoz S. 2011). El SIl constituye un 38\% en consultas más frecuentes con gastroenterólogos y el $12 \%$ de las consultas del médico general, siendo más común que la hipertensión arterial (10\%), el asma (4\%) y la enfermedad coronaria (2\%) (Klinger J. 2015). Debido a que es una enfermedad muy común produce altos costos en el consumo de medicamentos. Se le considera la segunda causa de ausentismo laboral después de la gripe.

\subsection{Tratamiento}

Se caracteriza por poseer tejidos para el almacenamiento de agua (Domínguez. R 2012).

Químicamente el Aloe vera se caracteriza por la presencia de constituyentes fenólicos que son generalmente clasificados en dos principales grupos: las hormonas, como la aloensina y las antraquinonas (libres y glicosiladas) como la barbaloína, isobarbaloina y la aloemodina; estos compuestos se encuentran en la capa interna de las células epidermales. La aloína es el principal componente del acíbar, que la planta secreta como defensa para alejar a posibles depredadores por su olor y sabor desagradable- (Domínguez. R 2012). También interviene en el proceso de control de la transpiración en condiciones de elevada insolación. La aloína es un glicósido antraquinónico que le confiere propiedades laxantes al acíbar (Tizard I. 2004).

El gel o pulpa es una masa gelatinosa e incolora formada por células parenquimatosas, estructuradas en colénquima y células pétreas delgadas (Vega. A 2005). EI gel está constituido principalmente de agua, mucílagos y otros carbohidratos, ácidos y sales orgánicas, enzimas, saponinas, taninos, heterósidos antracénicos, esteroles, triacilglicéridos, aminoácidos, ARN, trazas de alcaloides, vitaminas y diversos minerales.

\section{Materiales y Métodos}

-Diseño de estudio

La investigación realizada corresponde a un estudio de tipo observacional, descriptivo, horizontal y mixto.

-Tiempo, lugar y persona

El tiempo en que se realizó el estudio y aplicación de entrevistas, fue en octubre 2019.

El lugar donde se colectaron las entrevistas, fue en la escuela superior de Tepeji del Río.

Las personas incluidas en la investigación, fueron estudiantes de medicina de 1-4 semestre que oscilan de 18 a 25 años.

\section{-Criterios de selección}

Criterios de inclusión: estudiantes de la Licenciatura de médico cirujano de 1-4 semestre de la escuela superior de Tepeji del Río 
Criterios de exclusión: estudiantes que no están inscritos en la Licenciatura de médico cirujano, en la escuela superior de Tepeji del Río.

Criterios de eliminación: estudiantes de la Licenciatura de médico cirujano de 1-4 semestre de la escuela superior de Tepeji del Río, que no completaron la encuesta.

\section{-Población y muestra}

La Escuela Superior de Tepeji del Río cuenta con 1458 estudiantes, según las oficinas de dirección del plantel, los cuales están distribuidos en los tres niveles de bachillerato $64.5 \%$, licenciatura de médico cirujano $9.8 \%$, licenciatura en administración $11.8 \%$, licenciatura de ingeniería Industrial $13.9 \%$ de los estudiantes totales. De los cuales los estudiantes de medicina $9.8 \%$ oscila entre 18 y 25 años. Se aplicó un muestreo que se hizo para el estudio estadístico a conveniencia de la investigación, teniendo como resultado 136 estudiantes. El muestreo que se realizó para este estudio incluyó a los estudiantes de la Licenciatura de Médico Cirujano de la escuela superior de Tepeji del Río de los primeros 4 semestres.

El proceso que se siguió se ve reflejado en la figura 3 , donde se muestra el diagrama que ejemplifica los pasos realizados que se presenta a continuación.

1. Previo al estudio realizado sobre colitis nerviosa y su epidemiología a nivel nacional para todos los grupos de edad y/o género, se seleccionó a la población estudiantil (18-25 años) de 1-4 semestre de la licenciatura en médico cirujano de la escuela superior de Tepeji del Río, de acuerdo a los grupos con mayor desventaja debido a la influencia al estrés según estudios realizados.

2. Se realizó un boceto para las encuestas que posteriormente se les aplicó sólo a los estudiantes de medicina de dicha institución.

3. Los datos se recolectaron en el mes de octubre de 2019 en la escuela superior de Tepeji del Río.

4. El trabajo experimental se realizó dentro de la institución.

5. Se asistió a los 4 salones de medicina para dar a conocer la investigación a los estudiantes, con el objetivo de invitarlos a participar en nuestras encuestas.

6. Se aplicó una encuesta a los estudiantes de medicina para obtener información complementaria respecto a los síntomas que podrían presentarse si se tiene colitis nerviosa.

7. Con la información recabada, se realizó una base de datos para identificar la cantidad de estudiantes que presentan colitis nerviosa.

8. Se analizaron las variables que se midieron en la encuesta tales como: edad, género y sintomatología.

9. Partiendo de los resultados obtenidos, se comenzó con la elaboración de infusiones de Aloe vera.

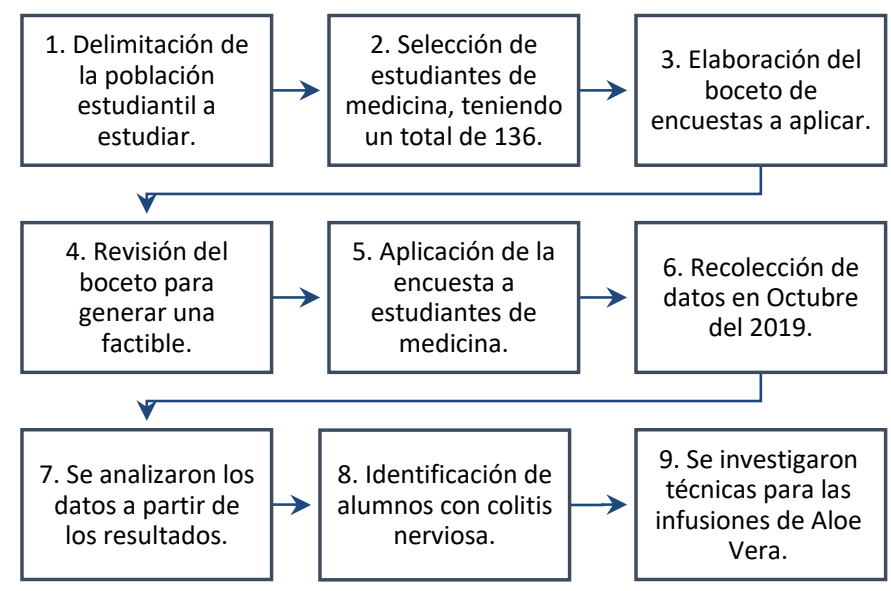

\section{Esquema SEQ Esquema_l*ARABIC 1} Metodología general

Figura 3. Proceso de selección y delimitación de la población estudiantil para implementar las infusiones de Aloe vera. Fuente: Elaboración propia, 2019.

\subsection{Procedimiento}

1. Se recomienda obtener la penca natural (figura 5), que no haya pasado por algún tipo de procesamiento.

2. Lavar y dejar escurrir durante $8 \mathrm{hrs}$, esto para liberar el Acíbar de la penca ya que este lo usa como protección de depredadores, es una sustancia amarillenta, irritativa y si se consume en altas dosis puede ser laxante.

3. Cortaremos la punta, las orillas y un poco de la base de la penca (Figura 6).

4. Con tu mano dominante, toma la penca y la abriremos cortando uno de los 2 lados de sus caras, de forma que quede expuesta la pulpa.

5. Retirar el gel con una cuchara, evitando si es que haya quedado Acíbar en la penca, que este se mezcle (Figura 7 y 8$)$.

6. La pulpa se puede triturar el Aloe vera directamente en 1 vaso de agua (Figura 9).

En la figura 4 Elaboración de la infusión de Aloe vera (Núñez. D 2015) se muestran los pasos previamente explicados a manera de resumen.

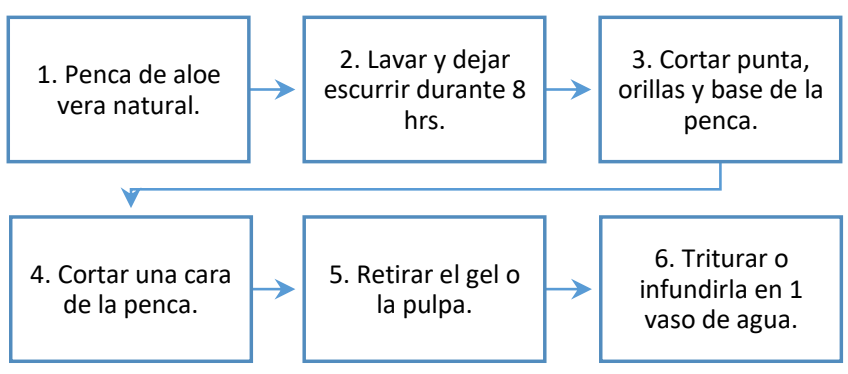

Figura 4. Elaboración de la infusión de Aloe vera (Núñez. D 2015). 


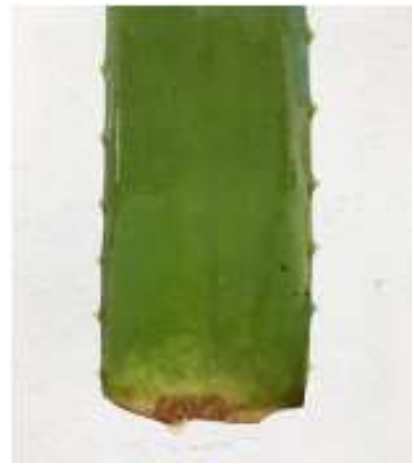

Figura 5. Penca de Aloe vera, lavada y dejada reposar durante 8 horas. Edición: propia, 2019.

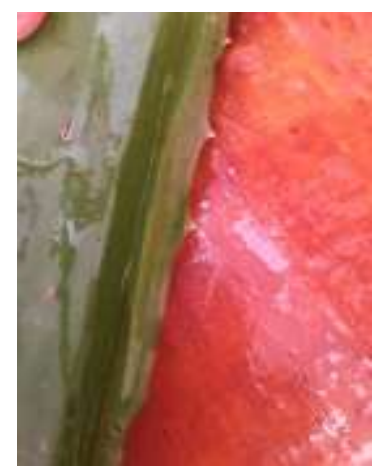

Figura 6. Corte vertical de una penca de Aloe vera. Edición: propia, 2019.

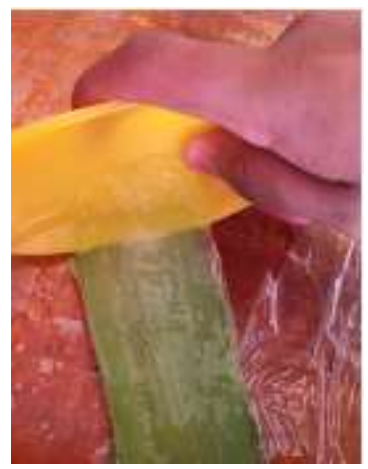

Figura 7. Extracción de la pulpa con una cuchara de plástico. Edición: propia, 2019.

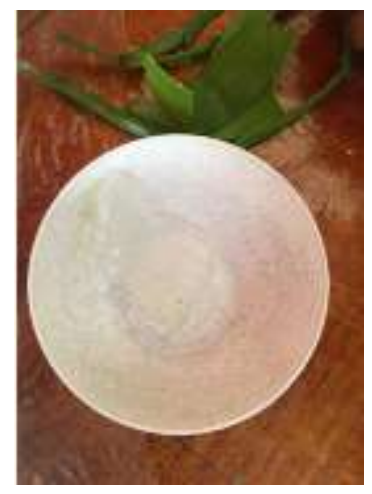

Figura 8. Recolección de la pulpa en un contenedor.
Edición: propia, 2019.

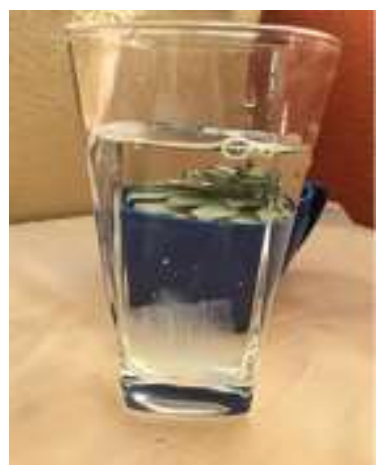

Figura 9. Infusión de Aloe vera. Edición: propia, 2019.

\subsubsection{Experimentación de infusiones de Aloe vera en estudiantes de medicina.}

A lo largo de la primera semana de noviembre de 2019 se llevó a cabo la experimentación de las infusiones de Aloe vera para el tratamiento de la colitis nerviosa, donde se tomó como muestra de población a diez estudiantes de medicina quienes presentaban el síndrome de intestino irritable. (Figura 10).

Tomando como precaución que la sábila al ser ingerida por primera vez, tiende a producir un poco de diarrea debido a que produce una limpia inicial en los intestinos, tanto de bacterias como de alimentos detenidos en pliegues o divertículos.
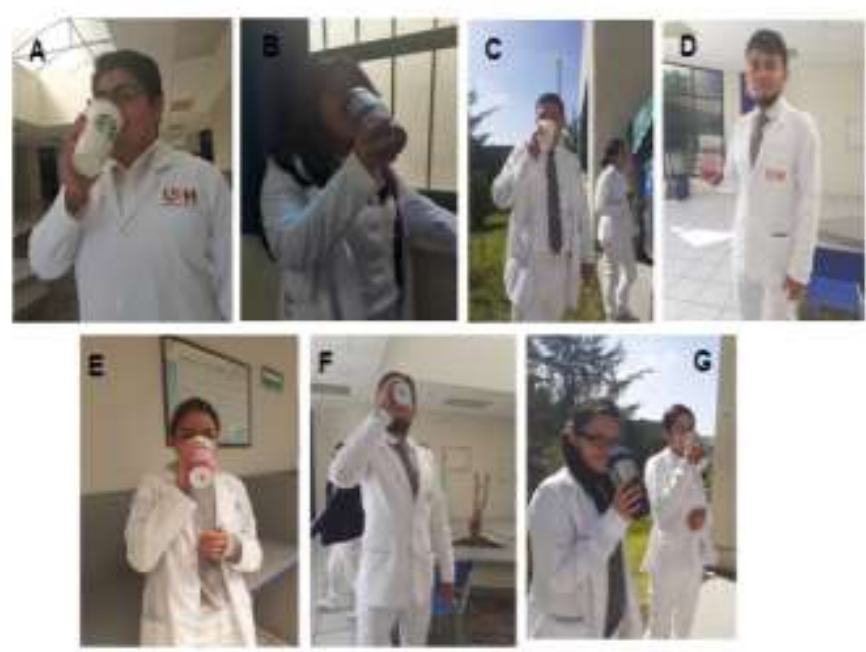

Figura 10. Estudiantes de la Licenciatura de Médico Cirujano probando la infusión de Aloe vera.

\section{Discusiones}

Examinando los resultados obtenidos por la encuesta y comparándolo con los fundamentos originales de la hipótesis del proyecto podremos discutir que: 
Tabla 2. Resultados obtenidos de las encuestas aplicadas

\begin{tabular}{|c|c|}
\hline & Personas encuestadas 136 \\
\hline & Edad 18-24 años \\
\hline $\begin{array}{l}61 \\
\text { hombres }\end{array}$ & 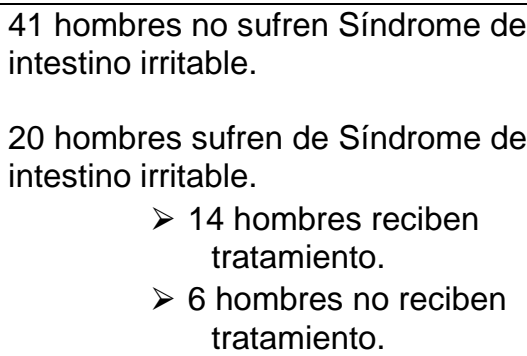 \\
\hline $\begin{array}{l}75 \\
\text { mujeres }\end{array}$ & $\begin{array}{l}36 \text { mujeres no sufren Síndrome de } \\
\text { intestino irritable. } \\
39 \text { mujeres sufren de síndrome de } \\
\text { intestino irritable. } \\
>28 \text { reciben tratamiento. } \\
>11 \text { no reciben tratamiento }\end{array}$ \\
\hline
\end{tabular}

La encuesta nos ayudó a corroborar como se muestra a continuación...

Tabla 3. Resultados

\begin{tabular}{|l|}
\hline \multicolumn{1}{|c|}{ Síntomas de riesgo presentados } \\
\hline Cólicos intestinales \\
Dolor abdominal
\end{tabular}

De los diferentes síntomas que experimentan los encuestados ocupan en 1er lugar los cólicos intestinales y dolor abdominal lo que quiere decir que muy probablemente sufren de colitis por problemas alimenticios y ayunos. Las dietas deben ser bajas en grasas y ricas en proteínas, así como evitar las comidas abundantes y pesadas, es preferible comidas más frecuentes y menos copiosas, evitar los alimentos que puedan desencadenar o empeorar los síntomas. Aquellos que con frecuencia perjudican son: especias, alcohol, sopas de sobres, cacao, pasteles, helados, mantequilla, derivados de lácteos, café, té, chocolate, bebidas con gas y alimentos flatulentos como: col, coliflor, habas, garbanzos, lentejas, cebollas, frutos secos y en conserva (Pérez. A, 2013).

Hablando de la alimentación, la encuesta mostró que al menos un $56 \%$ de los estudiantes padecían de inflamación intestinal después de consumir alimentos.

Tabla 4. Resultados del SII

\begin{tabular}{|c|c|}
\hline \multicolumn{2}{|c|}{$\begin{array}{l}\text { Inflamación Intestinal } \\
\text { (72 estudiantes) }\end{array}$} \\
\hline 24 hombres & 48 mujeres \\
\hline $\begin{array}{l}>\quad 17 \text { padecen del } \\
\text { SII }\end{array}$ & $\begin{array}{l}>32 \text { padecen } \\
\text { SIl }\end{array}$ \\
\hline $\begin{array}{l}>\quad 7 \text { no padecen } \\
\text { del SIl }\end{array}$ & $\begin{array}{l}16 \text { no } \\
\text { padecen } \\
\text { del SIl }\end{array}$ \\
\hline
\end{tabular}

Aunque algunos estudiantes niegan padecer el Síndrome de intestino irritable sufren ya de síntomas lo que condiciona en un futuro puedan padecerla. Recordemos que la encuesta fue contestada por alumnos, lo que puede justificarse el hecho de una alimentación desbalanceada y también de posibles ayunos lo que puede llevar a no padecer la enfermedad, pero sí tener síntomas que si no se tratan o se cambia la alimentación terminan por padecer el Síndrome de colon irritable.

En la encuesta, arrojó que cerca de 113 personas de las $136(83 \%)$ frecuentemente se exponen a situaciones de algún tipo de estrés, ansiedad y depresión ya sea en la escuela o fuera de ella.

Tabla 5. Resultados

\begin{tabular}{|c|}
\hline $\begin{array}{c}\text { Situaciones de algún tipo de estrés, } \\
\text { ansiedad y depresión } \\
113 \text { de } 136\end{array}$ \\
\hline $\begin{array}{c}54 \text { estudiantes padecen de SII } \\
59 \text { estudiantes no padecen SII }\end{array}$ \\
\hline
\end{tabular}

¿Qué quiere decir esto? Que al padecer o no colitis la mayoría de las personas viven con estrés continuo lo que es altamente riesgoso y que puede causar otros síntomas muy diferentes de la colitis. 99 personas mencionan haber tenido ayunos de más de 4 horas de lo normal de los cuales 50 de ellos padecen colitis y 49 a pesar de no tener colitis, tienen ayunos por trabajos, proyectos 0 exámenes escolares. Si cuestionamos qué personas son más susceptibles a la colitis nerviosa son las mujeres comparándolo con encuestas. De estas 136 personas experimentamos con 10 personas en infusiones de Aloe vera, de las cuales estuvieron de acuerdo para hacerlo. Está fundamentado que el Aloe vera tiene múltiples beneficios gastrointestinales, las personas que fueron tratadas con Aloe vera no solo sufrían de colitis nerviosa también padecían de problemas de estrés, pie de atleta, dolor muscular y dermatitis (Zawahri M, 2014). El tratamiento debe ser de 30 días y 1 semana de descanso, pero pese al tiempo hemos tomado resultados después de solo 1 semana, teniendo cambios favorables y satisfactorias para los estudiantes ya que como comentan no solo les ha ayudado a disminuir sus síntomas de colitis también les ha ayudado a otros problemas que presentaban, a pesar del tiempo el tratamiento seguirá ya que han notado buenos resultados, siguiendo con el tratamiento (Greedon G, 2015). El uso de fármacos para el tratamiento de la colitis nerviosa puede llevar a otros diferentes problemas por lo que, con lo obtenido se recomiendo eficaz y verazmente el uso del Aloe vera para su tratamiento, no solo es natural y menos costosa. También te lo agradecerá tu cuerpo (Hear. L, 2015). Como recomendaciones extra pueden acudir a ayuda psicológica en caso de que la colitis sea producto del estrés y de la ansiedad para una ayuda extra complementándolo con el Aloe vera así mismo tratar de cuidar la alimentación. 


\section{Conclusión}

En el trabajo anteriormente presentado se logró demostrar el funcionamiento del Aloe vera como tratamiento para el Síndrome del Intestino Irritable, basado en una investigación previa acerca de sus propiedades curativas de la planta referida, un estudio de campo apoyado de encuestas a los estudiantes de la Licenciatura en Médico Cirujano inscritos en la escuela Superior Tepeji del Río, con un total de 140 alumnos inscritos y basados en 136 entrevistados de los cuales 59 presentaron el Síndrome del Intestino Irritable, de estos solo diez aceptaron usar la infusión del Aloe vera, la cual fue administrada durante la primer semana de Noviembre del año 2019, una vez pasada la semana se encuestó a los diez estudiantes los cuales afirman que los síntomas del SII que presentaban fueron disminuidos en un $40 \%$, lo cual es insuficiente para el cumplimiento de la hipótesis previamente planteada, a lo cual se atribuye la limitación del tiempo de administración de las infusiones ya que originalmente deben ser treinta días de administración pero debido a la falta de tiempo fueron administradas durante siete días, sin embargo siete días bastaron para comprobar que las propiedades curativas del Aloe vera disminuyen los síntomas del Síndrome del Intestino Irritable.

\section{Referencias}

[1] Alvarado J. (2014) Convenio latinoamericana sobre el Síndrome del intestino

irritable: https://www.sciencedirect.com/science/article/pii/S0210570503704701

[2] Andrade A. (2017). El estrés y el sistema digestivo: https://idus.us.es/xmlui/bitstream/handle/11441/64735/GANFORNIN A\%20ANDRADES \%2c\%20ANA.pdf?sequence $=1 \&$ is Allowed=y

[3] Angiano A. (2016). Factores de estrés académico en estudiantes de licenciatura en enfermería durante las prácticas clínicas. Vol.3 Núm.1. https://www.rediech.org/ojs/2017/index.php/recie/article/view/260

[4] Bautista R., Ortiz R., Muñoz S. (2011). Síndrome de intestino irritable en estudiantes de medicina. Vol.4 Núm.3. http://www.scielo.org.mx/scielo.php?script=sci_arttext\&pid=S002617422011000300002

[5] Buenfil F. (2009). Aloe Vera: www.conabio.mx

[6] Carmona R. (2016) Convenido mexicano sobre el síndrome del intestino irritable:

https://www.sciencedirect.com/science/article/pii/S0375090616000185

[7] Castañeda R. (2014) Síndrome del intestino irritable trastorno crónico: https://www.elsevier.es/es-revista-medicina-universitaria-304-articulosindrome-intestino-irritable-X166557961050442X

[8] Díaz M. (2014) Recursos médicos para el Síndrome del intestino irritable:

[9] Domínguez R. (2012) El gel de aloe vera: estructura, composición química, procesamiento, actividad biológica e importancia en la industria farmacéutica y alimentaria. Rev. Mx. Ing.Quim. Vol. 11 México abr. 2012: http://www.scielo.org.mx/scielo.php?script=sci_arttext\&pid=S166527382012000100003

[10] Donaires N. (2015) Prevalencia y asociación de la dispepsia en el Síndrome del intestino irritable: http://www.scielo.org.pe/scielo.php?pid=S1022

$51292002000200003 \&$ script=sci_arttex
[11] Eshun K. (2004) Aloe Vera: A Valuable Ingredient for the Food, Pharmaceutical and Cosmetic Industries A Rev Citracal Reviews and Food Science Nutr 2004: http://www.scielo.org.pe/scielo.php?script=sci_arttext\&pid=S102251292005000200008

[12] Estrada L. (2018). Estrés académico en estudiantes de enfermería en Cartagena, Colombia. Vol. 40. https://revistas.javeriana.edu.co/index.php/imagenydesarrollo/article/vi ew/1645m7

[13] Ganfornina A. (2015) El estrés y el sistema digestivo: https://idus.us.es/xmlui/handle/11441/64735

[14] Gates G. (2016) Horticultura de aloe vera: https://www.botanicalonline.com/plantas-medicinales/aloe-vera-propiedades-caracteristicas

[15] Gómez M. (2016) Aloe vera y apoptosis en la colitis nerviosa: https://www.google.com/url?sa=t\&source=web\&rct=j\&url=https://ww w.medigraphic.com/pdfs/vertientes/vre-

2011/vre112a.pdf\&ved=2ahUKEwj14Z3AhtnlAhVUCjQIHR8WCrg4 ChAWMAN6BAgIEAE\&usg=AOvVaw2yZ2BDuwhX0JqyblYfqVxt

[16] González O. (2016) Dieta para el colon irritable: https://laguiadelasvitaminas.com/dieta-para-la-colitis/

[17] Greedon G. (2015) Propiedades y uso del aloe vera: https://www.ecoagricultor.com/propiedades-y-usos-del-aloe-vera/

[18] Hear L. (2015) Efectos del bajo peso molecular en los constituyentes del aloe vera: http://scielo.sld.cu/scielo.php?script=sci_arttext\&pid=S086403192006000300004

[19]Heggers J. (2016) Plantas medicinales: http://www.academia.edu/download/32125177/Curso_Fitoterapia_Ash ecova.pdf

[20]Hernández N. (2011). Universidad de Guadalajara: www.udg.mx./noticia

[21] Herrera L. (2016) Usos terapéuticos del Aloe vera: http://scielo.sld.cu/scielo.php?script=sci_arttext\&pid=S003475152016000100013

[22] Juárez. (2009). Boletín UNAM: https://www.dgcs.unma.mx

[23] Kallergis K. (2017) Afectaciones de la colitis nerviosa: https://www.google.com/amp/s/amp.elnuevoherald.com/vivirmejor/salud/article28451635.html

[24] Klinger J. (2015). Síndrome del intestino irritable. Scielo. Revista Médica de Chile: https://scielo.conicyt.cl/scielo.php?pid=S003498872001000500016\&script=sci_arttext

[25] Lawless. J. (2005) Aloe vera - Natural Wonder Cure. Harper Collins Publisher, Hammersmith, London, 2000: https://scholar.google.com/scholar?q=+Aloe+vera-

Nature $\% 3 F \% 3 F$ s+Gift\&as_sdt=0\&lr=\&hl=es\#d=gs_qabs\&u=\%23p $\%$ 3DVTGEyq-VC2gJ

[26] Mayo Clinic. (15 de agosto, 2019) Síndrome del Intestino Irritable: https://www.mayoclinic.org/es-es/diseases-conditions/irritable-bowelsyndrome/symptoms-causes/syc-

20360016?utm_source $=$ Google\&utm_medium $=$ abstract\&utm_content =Irritable-bowel-syndrome\&utm_campaign=Knowledge-panel

[27] Martínez R. (2013) Extracto del aloe vera: https://actualfruveg.com/2017/08/01/las-propiedades-del-aloe-veracomprobadas-cientificamente/

[28] Mearín F., Montoro M. (2011). Síndrome del Intestino irritable. 3era edición: https://www.aegastro.es/sites/default/files/archivos/ayudaspracticas/36_Sindrome_de_intestino_irritable.pdf

[29] Mendoza M. (2013) "Frecuencia de síndrome de intestino irritable en estudiantes de egreso de la licenciatura de médico cirujano de la facultad de medicina, universidad autónoma del estado de México. 2013" Toluca, Estado de México. 
http://ri.uaemex.mx/bitstream/handle/20.500.11799/13865/415096.pdf; jsessionid=8D89763AEEA2B4D58C4E2B1D4FFDF4EF? sequence $=1$

[30] Morales, P. (2012). Colitis Nerviosa: https://www.clikisalud.net/gastritis/la-colitis-que-es-y-cuales-son-sussintomas/

[31]Núñez D. (2015) Infusiones de aloe vera: https://repository.upb.edu.co/handle/20.500.11912/2017

[32] Ortiz J. (2016) Aloe vera la planta del futuro https://books.google.com.mx/books?hl=es\&lr=\&id=bWCB4uNQ1JcC $\&$ oi $=$ fnd \&pg $=$ PP $2 \& d q=$ colitis + nerviosa $+y+$ aloe + vera $\&$ ots $=X M C a Q N$ nfJ2\&sig=UMaza2LUPUHSOXVQiRDjkO3DCyU

[33] Otero W., Gómez M. (2015) SII: Diagnóstico y tratamiento farmacológico. Revista de Gastroenterología, Perú http://www.scielo.org.pe/scielo.php?script=sci_arttext\&pid=S102251292005000200008

[34] Panisello J. (2014) Nutrición y tratamiento de enfermedades de colon irritable: $\quad$ http://scielo.isciii.es/scielo.php?pid=S1139$76322011000400002 \&$ script=sci_arttext\&tlng=en

[35] Pérez A. (2013) Que comer cuando tienes colitis nerviosa: http://www.pmfarma.com.mx/noticias/3167-que-comer-cuando-sufresde-colitisnerviosa.html

[36] Pérez G. (2016) Complicaciones de la colitis nerviosa: https://scielo.conicyt.cl/scielo.php?pid=S003498872015000400015\&script=sci_arttext

[37]Ramírez G. (2013) Sábila: https://www.google.com/url?sa=t\&source=web\&rct=j\&url=https://dial net.unirioja.es/descarga/articulo/4956300.pdf\&ved=2ahUKEwiunu6j NjlAhUrjKOKHaAtAuoQFjAAegQIAhAB\&usg=AOvVaw2CNaXI3d gSkjJtmVJC0vtg

[38] Remes J. (2014) Tratamiento farmacológico del síndrome del intestino irritable: http://mixacademico.alianzamedicamexicana.com/medicoTransforma/ adminAM/forum/files_forum/122/41/41_Sindrome_de_intestino_irrita ble.pdf

[39] Richardson J. (2015) Eficiencia del aloe vera: https://actualfruveg.com/2017/08/01/las-propiedades-del-aloe-veracomprobadas-cientificamente/

[40] Smith J. (2013) Terapia para SII con aloe vera: https://www.mayoclinic.org/es-es/diseases-conditions/ulcerativecolitis/diagnosis-treatment/drc-20353331

[41] Tizard I. (2004) Isolation and characterization of structural components of Aloe vera Leaf pulp. International immunopharmacology 2004: http://www.scielo.org.mx/scielo.php?script=sci_arttext\&pid=S002617422011000300002

[42] Torres M. (2016) Etiología de la enfermedad de colon irritable:

https://core.ac.uk/download/pdf/159384254.pdf

[43] Vázquez B. (2016) Ingredientes del aloe vera en la farmacéutica:

https://www.google.com/url?sa=t\&source=web\&rct=j\&url=https://www.j dc.edu.co/revistas/index.php/Cult_cient/article/download/39/152/\&ved $=2$ ahUKEwiks46PhNnlAhXm0eAKHS3VBIM4ChAWMAd6BAgAE AE\&usg=AOvVaw2tO8sZoNcrhkApKIwK9ska

[44] Vega A (2005) Aloe vera como componente de alimentos funcionales. Rev. Chile. Nutr. Vol. 32, No3, Dic. 2005: https://scielo.conicyt.cl/scielo.php?script=sci_arttext\&pid=S071775182005000300005

[45]Villanueva E. (2016) Alimentos en la colitis nerviosa: https://amp.eluniversal.com.mx/amp/note/amp/eluniversal/1001409

[46] Zawahry M. (2014) Uso de aloe vera en el tratamiento de colitis nerviosa: https://www.google.com/amp/s/mejorconsalud.com/aloevera-para-tratar-la-colitis-nerviosa/amp/ 